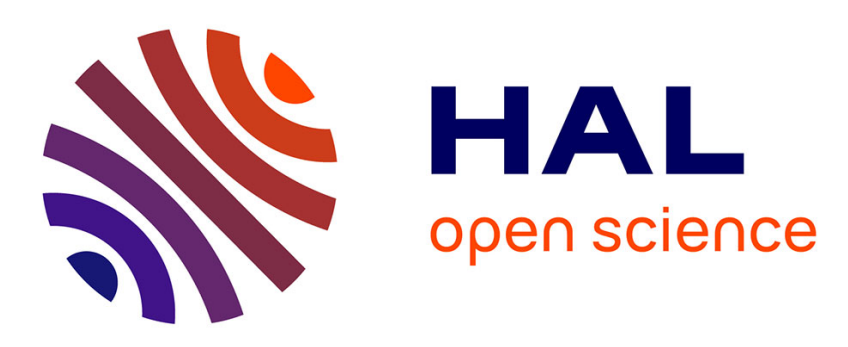

\title{
Les variations récentes de la ligne de rivage sur le littoral syrien
}

Rémi Dalongeville, Jacques Laborel, Paolo Pirazzoli, Paul Sanlaville, Maurice

Arnold, Paul Bernier, Jacques Evin, Lucien François Montaggioni, Lucien

Montaggioni

\section{To cite this version:}

Rémi Dalongeville, Jacques Laborel, Paolo Pirazzoli, Paul Sanlaville, Maurice Arnold, et al.. Les variations récentes de la ligne de rivage sur le littoral syrien. Quaternaire, 1993, 4 (1), pp.45 - 53. 10.3406/quate.1993.1990 . hal-03584290

\section{HAL Id: hal-03584290 \\ https://hal.science/hal-03584290}

Submitted on 22 Feb 2022

HAL is a multi-disciplinary open access archive for the deposit and dissemination of scientific research documents, whether they are published or not. The documents may come from teaching and research institutions in France or abroad, or from public or private research centers.
L'archive ouverte pluridisciplinaire HAL, est destinée au dépôt et à la diffusion de documents scientifiques de niveau recherche, publiés ou non, émanant des établissements d'enseignement et de recherche français ou étrangers, des laboratoires publics ou privés. 


\section{Les variations récentes de la ligne de rivage sur le littoral syrien}

Rémi Dalongeville, Jacques Laborel, Paolo Pirazzoli, Paul Sanlaville, Maurice Arnold, Paul Bernier, Jacques Evin, Lucien François Montaggioni

\section{Citer ce document / Cite this document :}

Dalongeville Rémi, Laborel Jacques, Pirazzoli Paolo, Sanlaville Paul, Arnold Maurice, Bernier Paul, Evin Jacques, Montaggioni Lucien François. Les variations récentes de la ligne de rivage sur le littoral syrien . In: Quaternaire, vol. 4, $\mathrm{n}^{\circ} 1$, 1993. pp. 45-53;

doi : https://doi.org/10.3406/quate.1993.1990

https://www.persee.fr/doc/quate_1142-2904_1993_num_4_1_1990

Fichier pdf généré le 27/10/2018 


\title{
Résumé
}

Le littoral syrien est situé au centre des côtes du Levant, mais il semble affecté d'une évolution originale au cours des six mille dernières années par rapport aux rivages situés au nord et au sud. $\mathrm{Si}$ la tectonique est bien le moteur principal des variations récentes de la ligne de rivage et si deux niveaux-repères sont bien présents, à $+1,20 \mathrm{~m}$ et à $+0,60 \mathrm{~m}$, les mouvements enregistrés ne sont probablement pas simples, positifs ou négatifs, et ne se réduisent pas à deux soulèvements d'une ampleur moyenne d'un demi-mètre comme cela semble avoir été le cas en Turquie et au Liban au cours de la même période. L'onginalité de la côte syrienne a été mise en évidence par l'application d'un protocole d'étude associant étroitement la géomorphologie des environnements sédimentaires et des substrats calcaires, la biologie des organismes constructeurs et la néotectonique.

\begin{abstract}
The coasts of Syna, located in the central part of the Levant, seem to have expenenced during the last 6,000 years an original evolution, somewhat different from that of the littoral more to the north or south. Tectonics are indeed the main cause of recent coastal changes and two raised shorelines mark reference levels at $+1.2 \mathrm{~m}$ and $+0.6 \mathrm{~m}$. However, vertical displacements might have been non unidirectional and more complex than two simple uplifts of the order of half a metre each, like those reported during the same period from nearby areas in Turkey and the Lebanon. Onginal features of the Syrian coast have been evidenced by a pluridisciplinary approach, associating observations on the geomorphology of sedimentary environments and of the limestone bedrock to data on the biological zonation of bioconstructions and to neotectonic events.
\end{abstract}




\title{
LES VARIATIONS RECENTES \\ DE LA LIGNE DE RIVAGE SUR LE LITTORAL SYRIEN
}

\author{
Rémi DALONGEVILLE*, Jacques LABOREL**, Paolo PIRAZZOLI***, \\ Paul SANLAVILLE*, Maurice ARNOLD****, Paul BERNIER*****, \\ Jacques EVIN***** et Lucien-François MONTAGGIONI******
}

RESUME

Le littoral syrien est situé au centre des côtes đu Levant, mais il semble affecté d'une évolution originale au cours des six mille dernières années par rapport aux rivages situés au nord et au sud. Si la tectonique est bien le moteur principal des variations récentes de la ligne de rivage et si deux niveaux-repères sont bien présents, à $+1,20 \mathrm{~m}$ et à $+0,60 \mathrm{~m}$, les mouvements enregistrés ne sont probablement pas simples, positifs ou négatifs, et ne se réduisent pas à deux soulèvements d'une ampleur moyenne d'un demi-mètre comme cela semble avoir été le cas en Turquie et au Liban au cours de la même période. L'originalité de la côte syrienne a été mise en évidence par l'application d'un protocole d'étude associant étroitement la géomorphologie des environnements sédimentaires et des substrats calcaires, la biologie des organismes constructeurs et la néotectonique.

Mots-clés: Syrie, Holocène, paléorivages, Vermets, beach-rock, néotectonique.

\section{ABSTRACT}

\section{LAST HOLOCENE SHORELINE CHANGES ALONG THE COASTS OF SYRIA}

The coasts of Syria, located in the central part of the Levant, seem to have experienced during the last 6,000 years an original evolution, somewhat different from that of the littoral more to the north or south. Tectonics are indeed the main cause of recent coastal changes and two raised shorelines mark reference levels at $+1.2 \mathrm{~m}$ and $+0.6 \mathrm{~m}$. However, vertical displacements might have been non unidirectional and more complex than two simple uplifts of the order of half a metre each, like those reported during the same period from nearby areas in Turkey and the Lebanon. Original features of the Syrian coast have been evidenced by a pluridisciplinary approach, associating observations on the geomorphology of sedimentary environments and of the limestone bedrock to data on the biological zonation of bioconstructions and to neotectonic events.

Key-words: Syria, Holocene, shorelines, Vermetids, beach-rock, neotectonics.

\section{INTRODUCTION}

Diverses observations (Sanlaville, 1976; Dalongeville et Sanlaville, 1980) ayant montré que les côtes de la Syrie présentaient des témoins de lignes de rivage supérieures à l'actuelle, une nouvelle mission a été organisée (1) pour retrouver ces témoins, les dater et établir éventuellement des corrélations avec des rivages holo- cènes émergés de régions avoisinantes: le Liban (Sanlaville, 1977), la Turquie dans les régions d'Antalya et d'Alanya (Dalongeville et Sanlaville, 1977, 1979; Kelletat et Kayan, 1983) et plus près de la Syrie, au débouché de l'Oronte (Pirazzoliet al., 1991), voire d'autres régions de la Méditerranée orientale (Pirazzoli, 1986).

Les côtes syriennes sont carbonatées, à l'exception de quelques affleurements de basalte et d'ophiolites, près

* GREMO-URA 913, Université de Lyon 2-C.N.R.S., Maison de l'Orient, 7 rue Raulin, 69007 LYON.

** Laboratoire de Biologie Marine et d'Ecologie du Benthos, Département des Sciences de la Mer, Luminy, case 901, 13288 MARSEILLE Cedex9.

*** URA 141 C.N.R.S., Laboratoire de Géographie Physique, 1, Place Aristide Briand, 92190 MEUDON-BELLEVUE.

**** C.N.R.S.-C.E.A., Centre des Faibles Radioactivités, Avenue de la Terrasse, 91198 GIF-SUR-YVETTE.

**** URA 11, Université Claude Bernard-C.N.R.S., Centre des Sciences de la Terre, $43-46$ Bd du 11 novembre 1918, 69622 VILLEURBANNE Cedex.

****** URA 1208, Université de Provence-C.N.R.S., 13331 MARSEILLE. 
de la frontière turque et dans le centre de la façade maritime, à Banyas (fig. 1). Elles sont basses, sauf dans certains secteurs de falaises vives et plongeantes (à $R$ as al-Bassit, par exemple), et parfois rocheuses; elles correspondent alors à des affleurements de calcaires d'âge miocène ou pléistocène aplanis par l'érosion marine et continentale, comme dans la région de Lattaquieh où elles dessinent des caps plus ou moins avancés dans la mer. Assez souvent, elles sont meubles et correspondent à de vastes épandages fluviatiles et marins de sables et de galets. Mais le recul récent de ces côtes meubles a mis au jour des beach-rocks (Dalongeville et Sanlaville, 1984) qui résistent à l'érosion de sorte que les portions de côtes montrant de véritables plages sont rares en Syrie, même dans la baie du Akkar.

Le littoral présente un certain nombre de témoins de variations récentes de la ligne de rivage: sédiments de plage indurés ou non, formes d'érosion du substrat dur (trottoirs, encoches), constructions organogènes (biostromes à Vermets). Tous ces témoins n'ont ni la même signification ni la même fiabilité, mais ils sont liés à des niveaux marins différents de l'actuel. Les relais qui s'opèrent dans le temps et dans l'espace entre dépôts de plage et constructions organogènes permettent d'avoir une vision assez précise des événements morphologiques et hydrodynamiques qui se sont succédé au cours des six derniers millénaires.

Un premier épisode est marqué, à $+1,20$ m environ, mais il n'en reste que peu de témoins caractéristiques. Un deuxième niveau, à $+0,60 \mathrm{~m}$, peut se suivre sur l'ensemble du littoral.

\section{METHODES}

L'étude porte essentiellement sur les secteurs où existent des vestiges non seulement significatifs par leur

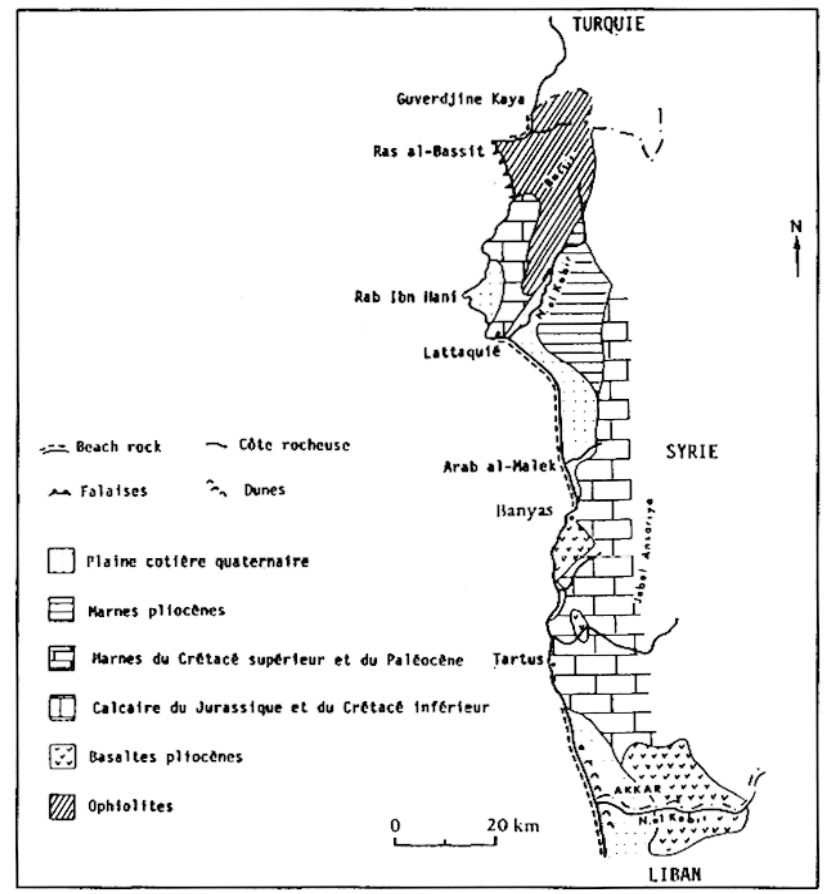

Fig. 1: Carte du littoral syrien. Localisation des principaux points d'observation cités dans le texte.

Fig. 1: Map of the Syrian coast and location of the main sites mentioned in the tex. modelé et leur faciès mais encore datables par le radiocarbone. Deux types de supports ont été utilisés pour les datations: les constructions organogènes (biostromes à Vermets) et les coquilles contenues dans les sédiments de plage consolidés ou non. Seules des datations sur des matériaux supposés indicateurs d'anciens rivages ont été effectuées (tab. I).

Les échantillons récoltés ont été répartis en deux catégories:

- ceux dont le poids était suffisant pour une analyse par comptage de radioactivité $14 \mathrm{C}$ ont été datés au Centre de Datation par le Radiocarbone de l'Université ClaudeBernard - Lyon 1 (références de labo: Ly-);

- ceux de petite ou très petite taille pour lesquels la technique dite «A.M.S.» de couplage d'accélérateur et de spectrométrie de masse était nécessaire ont été mesurés au Tandétron du Centre des Faibles Radioactivités de Gif-sur-Yvette (références de labo: Gif-AMS).

Afin de comparer entre eux les résultats obtenus par les deux méthodes d'analyse, tous les âges conventionnels (exprimés en BP) ont été calculés après une normalisation à un «delta» $13 \mathrm{C}$ de $-25 \%$ PDB. La mesure du «delta» $13 \mathrm{C}$ de chaque échantillon a été systématiquement effectuée pour les mesures au Tandétron. Elle n'a pas été faite pour les échantillons mesurés à Lyon mais seulement estimée à $0 \%$ PDB. La conversion de ces dates 14C BP en années réelles a été faite à l'aide de la courbe de calibration des échantillons marins proposée par Stuiver et al. (1986), en prenant pour la Méditerranée la valeur $R=-80 \pm 25$ (Stiros et al., 1991). Cette valeur revient à supposer que l'eau de mer superficielle a, près des côtes syriennes, un âge apparent de $320 \pm 25$ ans, mais il s'agit d'une estimation à partir de quelques données de la Méditerranée occidentale ou centrale et aucun chiffre précis n'a encore été obtenu dans la région étudiée ici.

Les marges données avec les dates exprimées en calendrier 14C BP (avant 1950) correspondent au critère de 1 sigma. Par contre, les plages de temps en années réelles exprimées en années avant/après J.-C. correspondent à la probabilité de $95 \%$ ( 2 sigmas), suivant les conventions internationales.

\section{LES TEMOINS DE VARIATIONS DE LA LIGNE DE RIVAGE}

Les résultats ne sont pas toujours présentés de la même manière dans le texte: tantôt, en effet, nous avons privilégié le lieu d'observation, tantôt le type de support d'observation.

\section{LE NIVEAU DE $+1,20 \mathrm{M}$}

Guverdjine Kaya: les formes de corrosion et de construction organogène

A Guverdjine Kaya (fig. 2), des placages de Vermets (Dendropoma petraeum) ont été observés à 1,20 m (1,2 $\pm 0,3 ;$ tab. I). Les restes du biostrome ne présentent pas d'altération, même si la construction organogène a été quelque peu retouchée par une abrasion ultérieure. Le biostrome recouvre partiellement un trottoir de corrosion d'une cinquantaine de centimètres de large taillé dans des calcaires du Crétacé, résistants et très redressés. A l'arrière, le trottoir passe à une encoche peu

(1) Cette étude résulte d'observations de terrain effectuées par quatre d'entre nous (R.D., J.L., P.A.P. et P.S.) le long du littoral syrien, de la frontière turque à la frontière libanaise, au cours de la première semaine de septembre 1991, dans le cadre du Programme EPOCH de la Commission des Communautés Européennes. 


\begin{tabular}{|c|c|c|c|c|c|c|c|c|c|c|}
\hline \multirow{2}{*}{$\begin{array}{c}\text { Echantillon } \\
n^{\circ}\end{array}$} & \multicolumn{4}{|c|}{ Localitè } & \multirow{2}{*}{$\begin{array}{l}\text { Matériel } \\
\text { (a) }\end{array}$} & \multirow{2}{*}{$\begin{array}{l}\text { Altitude } \\
\text { (m au-dessus } \\
\text { de } 1 \text { 'équivalent } \\
\text { actuel) }\end{array}$} & \multirow{2}{*}{$\begin{array}{l}\text { Position de } \\
1 \text { 'ancien } \\
\text { niveau moyen } \\
\text { de la mer } \\
\text { (m au-dessus } \\
\text { de } 1 \text { 'actuel) }\end{array}$} & \multirow{2}{*}{ Laboratoire } & \multicolumn{2}{|l|}{ Datations } \\
\hline & Nom & & $\begin{array}{l}\text { Lat. } \\
\mathrm{N}\end{array}$ & $\begin{array}{l}\text { Long. } \\
\text { E }\end{array}$ & & & & & $\begin{array}{l}\text { Age 14C } \\
\text { conventionne } 1 \\
(e) \\
\text { ans } B P \pm 10\end{array}$ & $\begin{array}{l}\text { Age calibré } \pm 2 \sigma(f) \\
\text { ans réels }\end{array}$ \\
\hline $91 \mathrm{SY} 5$ & Guverdjir & ine Kaya & $35^{\circ} 55^{\prime}$ & $35^{\circ} 54^{\circ}$ & D & $+0.6 \pm 0.2(b)$ & $+0.6 \pm 0.2$ & Ly -5623 & $1890 \pm 55$ & 280-580 ap. J. -C. \\
\hline 91 sy6 & , & . & $35^{\circ} 55^{\circ}$ & $35^{\circ} 54^{\circ}$ & D & $+1.2 \pm 0.3(b)$ & $+1.2 \pm 0.3$ & Ly -5624 & $5595 \pm 85$ & 4330-3950 av. J. -C. \\
\hline $\begin{array}{l}\text { AKKRA5-A } \\
\text { AKKRA5-B }\end{array}$ & , & , & $35^{\circ} 55^{\circ}$ & $35^{\circ} 5^{\circ}$ & $\mathrm{Cc}$ & $+2.5 \pm 0.3(c)$ & $\begin{array}{l}>+0.7, \leq+2.8 \\
>+0.7, \leq+2.8\end{array}$ & $\begin{array}{l}\text { Gif-AMS } \\
\text { Gif-AMS }\end{array}$ & $\begin{array}{l}3590 \pm 70 \\
2945 \pm 55\end{array}$ & $\begin{array}{l}1820-1440 \text { av. J.-C. } \\
970-760 \text { av. J. -C. }\end{array}$ \\
\hline $91 \mathrm{SY} 4$ & Ras al $\mathrm{B}$ & Bassit & $35^{\circ} 51^{\circ}$ & $35^{\circ} 48^{\circ}$ & D & $+0.65 \pm 0.1(b)$ & $+0.65 \pm 0.2$ & $\begin{array}{l}\text { Ly- } 5622 \\
\text { Gif-AMS } \\
\text { Gif-AMS }\end{array}$ & $\begin{array}{l}1335 \pm 50 \\
1450 \pm 60 \\
1400 \pm 70\end{array}$ & $\begin{array}{l}890-1100 \text { ap. J.-C. } \\
730-1020 \text { ap. J.-C. } \\
770-1060 \text { ap. J.-C. }\end{array}$ \\
\hline $91 \mathrm{sY} 1$ & Ras Ibn & Hani & $35^{\circ} 35^{\circ}$ & $35^{\circ} 44^{\circ}$ & D & $+0.5 \pm 0.2(b)$ & $+0.5 \pm 0.2$ & Ly-5621 & $2590 \pm 45$ & $520-320$ av. J. $-\mathrm{C}$. \\
\hline $91 \mathrm{SY} 2$ & $\cdots$ & , & $35^{\circ} 35^{\circ}$ & $35^{\circ} 44^{\circ}$ & $\mathrm{Cm}$ & $+0.3(c)$ & $z+0.3$ & Ly -5636 & $3225=55$ & 1370-1010 av. J. -C. \\
\hline $91 \mathrm{SY7}$ & . & , & $35^{\circ} 35^{\circ}$ & $35^{\circ} 44^{\circ}$ & D & $+0.05 \pm 0.2(c)$ & \pm 0 & Ly -5625 & $1675 \pm 55$ & 550-770 ap. J.-C. \\
\hline $91 \mathrm{sy} 8$ & $\cdot$ & , & $35^{\circ} 35^{\circ}$ & $35^{\circ} 44^{\circ}$ & $\mathrm{Cm}$ & $+0.4 \pm 0.2(c)$ & $2+0.4$ & Ly -5637 & $2915 \pm 50$ & $910-750$ av. J. $-\mathrm{C}$. \\
\hline $\begin{array}{l}91 \mathrm{SY} 9 / 1 \\
91 \mathrm{SY} 9 / 2\end{array}$ & $\begin{array}{l}\cdots \\
\cdots\end{array}$ & $\begin{array}{l}\because \cdot \\
\cdots\end{array}$ & $\begin{array}{l}35^{\circ} 35^{\circ} \\
35^{\circ} 35^{\circ}\end{array}$ & $\begin{array}{l}35^{\circ} 44^{\circ} \\
35^{\circ} 44^{\circ}\end{array}$ & $\begin{array}{l}\mathrm{c} \\
\mathrm{c}\end{array}$ & $\begin{array}{l}+0.45(d) \\
+0.9(d)\end{array}$ & $\begin{array}{l}+0.80 \pm 0.75 \\
+1.25 \pm 0.75\end{array}$ & $\begin{array}{l}\text { Ly }-5626 \\
\text { Ly-5627 }\end{array}$ & $\begin{array}{l}1920 \pm 55 \\
1970 \pm 55\end{array}$ & $\begin{array}{l}250-550 \text { ap. J. -C. } \\
200-470 \text { ap. J. }- \text { C. }\end{array}$ \\
\hline $\begin{array}{l}91 \mathrm{SY} 15-\mathrm{A} \\
91 \mathrm{SY} 15-\mathrm{B}\end{array}$ & Arab al & Malek & $35^{\circ} 16^{\circ}$ & $35^{\circ} 55^{\circ}$ & D & $+0.6 \pm 0.1(b)$ & $+0.6 \pm 0.2$ & $\begin{array}{l}\text { Gif-AMS } \\
\text { Gif-AMS }\end{array}$ & $\begin{array}{l}1820 \pm 60 \\
1880 \pm 70\end{array}$ & $\begin{array}{l}370-650 \text { ap. J. -C. } \\
260-620 \text { ap. J. -C. }\end{array}$ \\
\hline $\begin{array}{l}91 \mathrm{SY} 16-\mathrm{A} \\
91 \mathrm{SY} 16-\mathrm{B} \\
91 \mathrm{SY} 16-\mathrm{C}\end{array}$ & , & , & $35^{\circ} 16^{\prime}$ & $35^{\circ} 55^{\circ}$ & D & $+0.4 \pm 0.1(b)$ & $+0.4 \pm 0.2$ & $\begin{array}{l}\text { Gif-AMS } \\
\text { Gif-AMS } \\
\text { Gif-AMS }\end{array}$ & $\begin{array}{l}2010 \pm 50 \\
1890 \pm 60 \\
1900 \pm 50\end{array}$ & $\begin{array}{l}150-430 \text { ap. J. -C. } \\
270-590 \text { ap. J. -C. } \\
280-560 \text { ap. J.-C. }\end{array}$ \\
\hline MALEK1 & , & . & $35^{\circ} 16^{\circ}$ & $35^{\circ} 55^{\circ}$ & $\mathrm{Cd}$ & $+1.5 \pm 0.2(c)$ & $<+1.5$ & Ly -5630 & $5960 \pm 60$ & 4680-4360 av. J.-C. \\
\hline $\begin{array}{l}\text { MALEK } 2 \mathrm{a} \\
\text { MALEK } 2 \mathrm{~b}\end{array}$ & : & $\because$ & $\begin{array}{l}35^{\circ} 16^{\circ} \\
35^{\circ} 16^{\circ}\end{array}$ & $\begin{array}{l}35^{\circ} 55^{\circ} \\
35^{\circ} 55^{\circ}\end{array}$ & $\begin{array}{l}V(r) \\
C\end{array}$ & $\begin{array}{l}+1.3 \pm 0.2(c) \\
+1.3 \pm 0.2(c)\end{array}$ & $\begin{array}{l}z+0.9, s+1.5 \\
z+0.9, \leq+1.5\end{array}$ & $\begin{array}{l}\text { Ly }-5631 \\
\text { Ly }-5632\end{array}$ & $\begin{array}{l}5275 \pm 65 \\
5155 \pm 65\end{array}$ & $\begin{array}{l}3940-3630 \text { av. J. }- \text { C. } \\
3780-3500 \text { av. J.-C. }\end{array}$ \\
\hline
\end{tabular}

(a): $D=$ Dendropoma; $C=$ coquilles marines $; C=$ coquilles marines dans conglomérat; $C d=$ coquilles marines dans des sédiments contenant des coquilles d'eau douce: $\mathrm{Cm}=$ ciment marin; $\mathrm{S}=$ Serpulorbis; $\mathrm{V}=$ Vermets; $\mathrm{Ac}=$ algues calcaires. $(\mathrm{r})$ : remanié.

(b): par rapport au niveau instantané de la mer lors du prélèvement. (c): au-dessus du niveau moyen de la mer actuel. (d): au-dessus de la nappe d'eau.

(e): âges normalisés pour $\delta 13 \mathrm{C}=-25 \%$ PDB. (f): en utilisant la courbe de calibration pour les échantillons marins proposee par stuiver et al. (1986) et en prenant pour la Méditerranee $\Delta_{R}=-80 \pm 25$ (Stiros et al., 1992).

Tab. I: Reconstitution des variations relatives récentes du niveau de la mer en Syrie. 


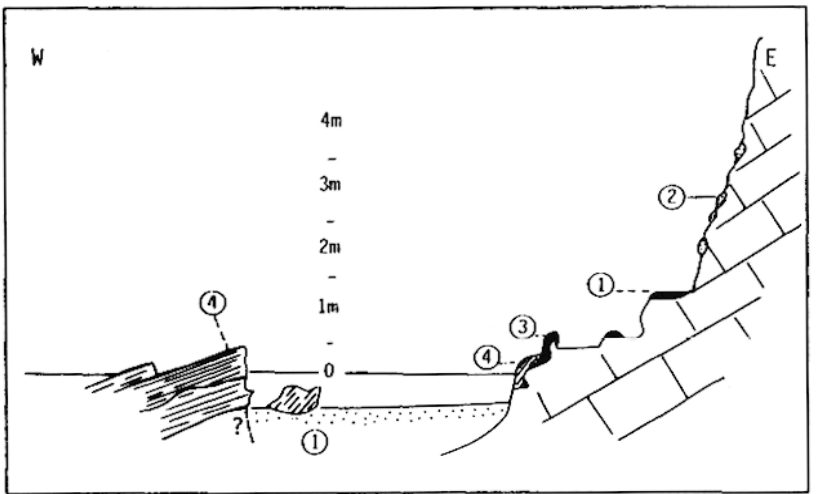

Fig. 2: Profnl littoral de la baie de Guverdjine Kaya (sud). 4 - Faciès littoraux d'une plage ancienne cimentée, conservés sous la forme d'un beach-rock et ayant érodé les Vermets à $+0,60 \mathrm{~m} .3$ - Biostrome a Vermets (daté de $1890 \pm 55 \mathrm{BP}$ ), associé à un trottoir de corrosion et à des cavités du type cuvette. Niveau de $+0,60 \mathrm{~m}$. 2 - Placages de sédiments de plage cimentés à la surface de la falaise (datés de 3590 $\pm 70 \mathrm{BP}$ à $2945 \pm 55 \mathrm{BP}$ ) et correspondant à un niveau marin indéterminé $(+1,20 \mathrm{~m}$ ) $) 1$ - Biostrome à Vermets (daté de $5595 \pm 85$ BP). Niveau de $+1,20 \mathrm{~m}$.

Fig. 2: Coastal profile in the south part of Guverdjine Kaya Bay. 4Litoral facies of a cemented ancient beach preserved as a beach-rock, having abraded the Vermetids at $+0,60 \mathrm{~m}$. 3 - Vermetid biostrome (dated $1890 \pm 55$ yr BP) associated with a surf bench and to pools: + $0,60 \mathrm{~m} .2$ - Veneer of beach deposits cemented on the cliff (dated from $3590 \pm 70$ to $2945 \pm 55 \mathrm{yr} B P$ ), corresponding to an undetermined sea level ( $1.2 \mathrm{~m}$ ?). 1 -Vermetid biostrome (dated $5595 \pm 85$ yr BP): +1.2 $m$ level.

profonde, assez ouverte, que l'on peut suivre plus au nord, courant le long de la falaise. Les Vermets et le trottoir attestent un épisode durablement stable de la mer à $+1,20 \mathrm{~m}$. Les Vermets sont datés de $5595 \pm 85 \mathrm{BP}$ (tab. I).

\section{Arab al-Malek: les dépôts de plage}

Le secteur d'Arab al-Malek a fourni des séquences sédimentaires particulièrement significatives. Une coupe de plus de $3 \mathrm{~m}$ de haut, levée à l'est de la piste littorale, montre une succession de deux séquences au moins (fig. 3).

La partie inférieure de la coupe est occupée par un sédiment siliciclastique rouge sombre [1a]. Vers le haut, on passe insensiblement à un dépôt [1b] dans lequel des coquilles terrestres (Helix) et marines (Murex, Cardium) sont mêlées. Il s'agit là d'un dépôt deltaïque, situé nettement au-dessus du niveau marin actuel puisque sa base se tient $\mathbf{a}+1,50 \mathrm{~m}$ et que son sommet est $\mathbf{a}+2,50$ $\mathrm{m}$. Les coquilles marines ont été datées de $5960 \pm 60 \mathrm{BP}$ (tab. I).

Le dépôt supérieur [2] est un sédiment marin qui monte jusqu'à plus de $4 \mathrm{~m}$ d'altitude absolue. Il ravine la formation deltaique en y taillant de profonds chenaux. Les grosses coquilles de Murex (datées de $5155 \pm 65 \mathrm{BP}$ ), les blocs de Vermets (datés de $5275 \pm 65 \mathrm{BP}$ ) et les galets correspondent à un cordon de plage, donc à l'étage supralittoral [2a]. Le démaigrissement de ces dépôts restés meubles a atteint le beach-rock très dur [2b] qui s'étend sur $20 \mathrm{~m}$ de large, en avant de la coupe et jusqu'au trait de côte actuel.

Ces deux dépôts, en particulier la deuxième formation, ne peuvent être raccordés à des niveaux marins précis; mais ils correspondent à une mer située nettement au-dessus du niveau actuel et l'on peut considérer que l'on a là des témoins multiples d'un même niveau, situé vers $+1,20 \mathrm{~m}$.
D'autres secteurs du littoral syrien n'ont livré que des observations plus fragmentaires. A Tell Soukkas, des Vermets à $+1,20 \mathrm{~m}$ sont peu développés et fortement attaqués par la corrosion supralittorale (embruns, paquets de mer et mazout).

Ces formes sont les marques précises, bien que dispersées et souvent endommagées, d'un niveau stable situé vraisemblablement à $+1,20 \mathrm{~m}$.

\section{LE NIVEAU DE + 0,60 M}

En de nombreux points du littoral syrien, des formes de corrosion du type trottoir et des biostromes à Dendropoma petraeum ont été conservés à une altitude oscillant le plus souvent entre $0,50 \mathrm{~m}$ et $0,80 \mathrm{~m}$ au-dessus de la limite supérieure des formes homologues actuelles. Les constructions de Vermets sont peu altérées, leur structure interne est homogène et ne montre ni stade d'érosion ni reprise de croissance.

Les datations donnent des âges compris pour la plupart entre $2010 \mathrm{BP}$ et $1820 \mathrm{BP}$.

\section{Guverdjine Kaya}

Un biostrome à Vermets se présente à $+0,60 \mathrm{~m}$, juste au-dessous de celui décrit précédemment à $+1,20 \mathrm{~m}$ (fig. 2). Il a été daté de $1890 \pm 55$ BP. Les Vermets sont associés à un profil littoral typique de l'étage médiolittoral en roches carbonatées: trottoir de corrosion $(1 \mathrm{~m}$ de large au maximum), encoche, cuvette. Un profil semblable est observable un peu plus au nord, en bordure de la baie voisine. Dans les deux cas, Vermets et formes de corrosion ont été recouverts d'un sable foncé qui est actuellement cimenté et dont il ne reste aujourd'hui que des placages. Il correspond à une plage différente de l'actuelle qui a fossilisé la falaise, postérieurement à l'établissement des Vermets à $+0,60 \mathrm{~m}$.

L'épais beach-rock qui subsiste en avant de la falaise et de la plage actuelle correspond probablement à cet épisode marin mais il n'a pas pu être daté, faute d'éléments coquilliers.

Les dalles du beach-rock sont les témoins d'un niveau marin supérieur à l'actuel de quelques décimètres $(0,60$

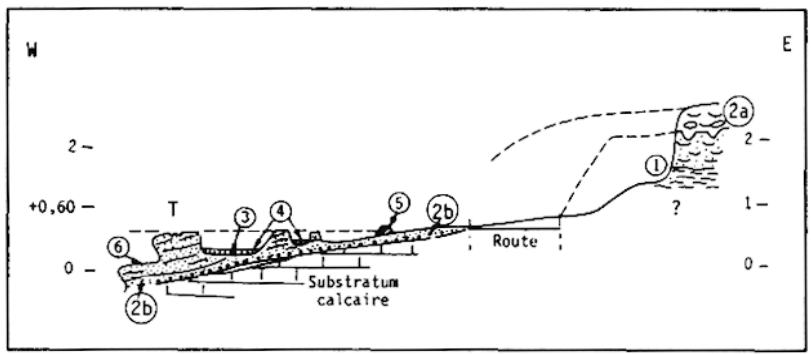

Fig. 3: Profil synthétique du littoral de Arab al-Malek. Formes de corrosion, formes de dépôt (deux épisodes) et Vermets, 6 - Trottoir de corrosion actuel. 5 - Biostrome à Vermets (daté de $2010 \pm 50$ BP à $1820 \pm 60$ BP). 4 - Excavations de carrières. 3 - Couche 3. Sédiments de plage récents non datés, peut-être responsables de la mort des Vermets du niveau de $+0,60 \mathrm{~m}$. 2 . Couche 2 (datée de $5275 \pm 65 \mathrm{BP}$ à $5225 \pm 65$ BP). 2a. Cordon de haut de plage (supralittoral), non cimenté. 2b. Faciès médiolittoral, cimenté sous la forme d'un beachrock 1 - Couche 1. Sédiments terrigènes à la base passant vers le sommet à des dépôts deltaĩques (datés de $5960 \pm 60$ BP).

Fig. 3: Simplified coastal profile at Arab al-Malek. Sea-corrosion feaures (two episodes) and Vermetids. 6 - Present surf bench. 5 . Vermetid biostrome, dated from $2010 \pm 50$ to $1820 \pm 60$ yr BP. 4 Quamy. 3 - Formation 3, undated recent beach sediments, which might have killed the Vermetids. 2. Formation 2, dated $5275 \pm 65$ and 5155 $\pm 65 \mathrm{yr}$ BP; $2 a$ - upper beach (supralittoral) deposits; $2 b$ - mediolitoral facies cemented as a beach-rock 1 - Formation 1 : temigenous deposits (below) capped by delta deposits (above), dated $5960 \pm 60$ yr BP. 


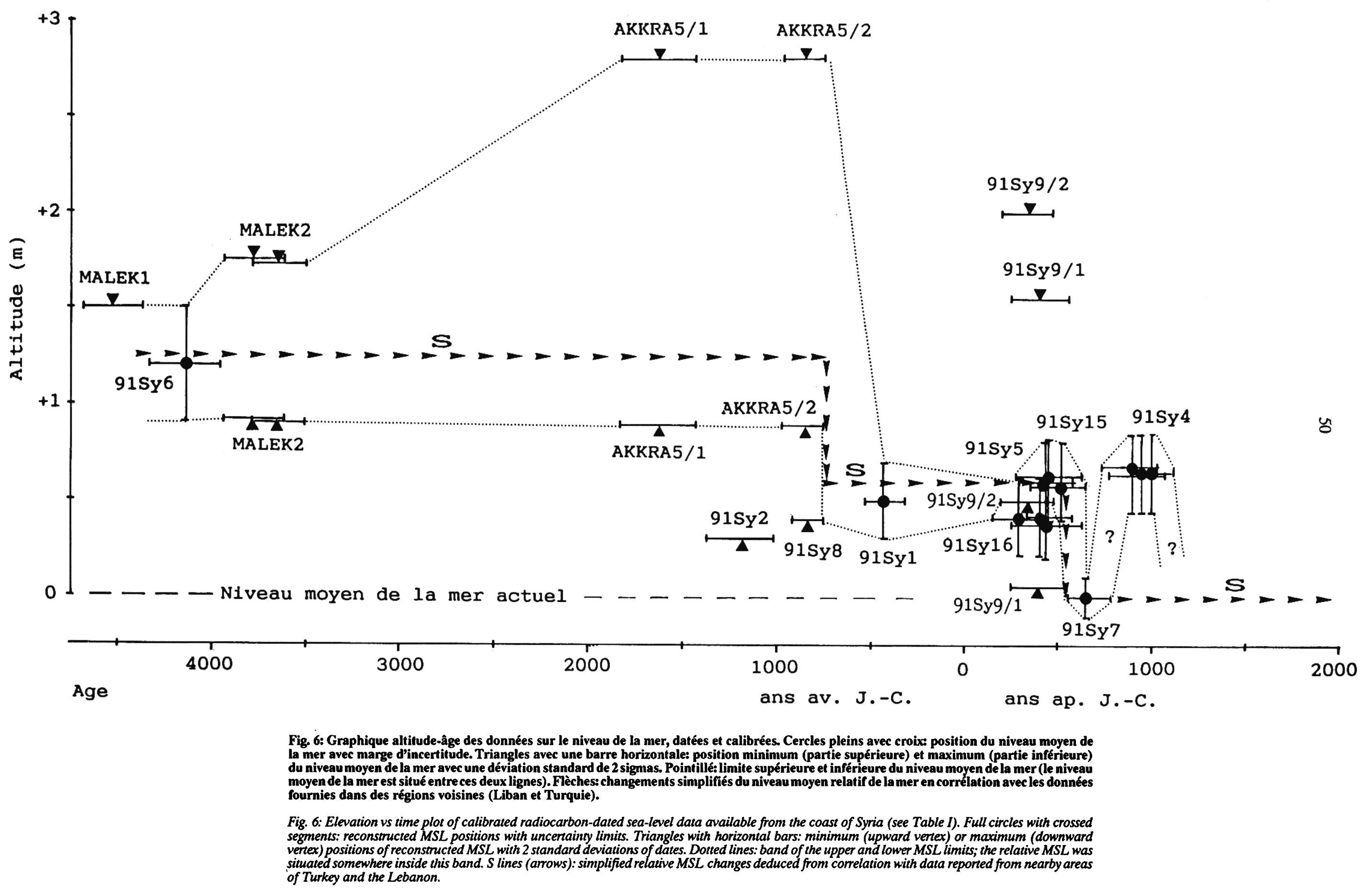




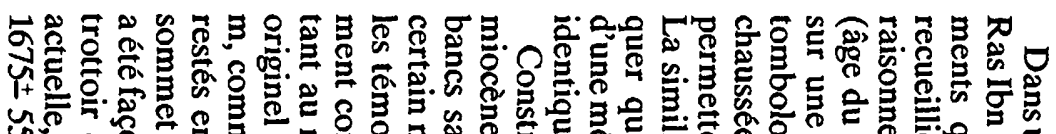

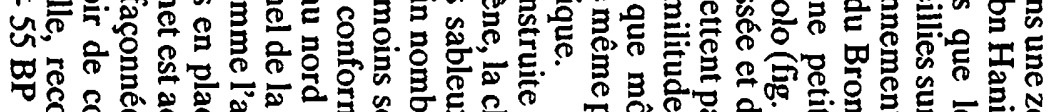

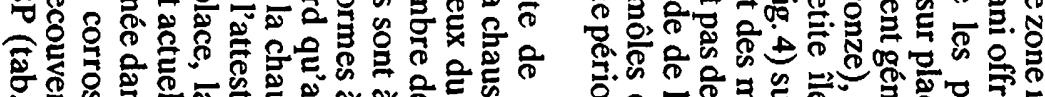

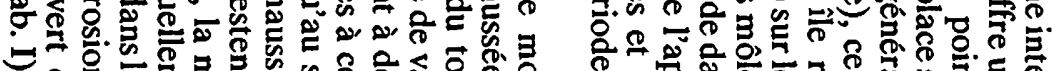

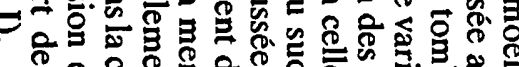

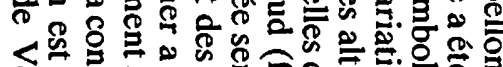

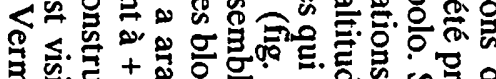

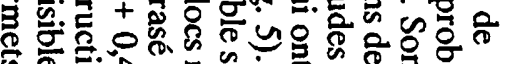
के 证.

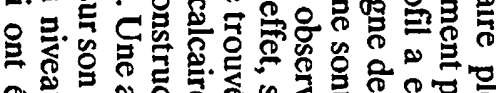

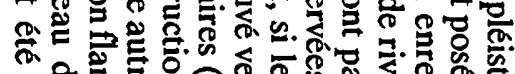

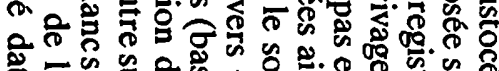

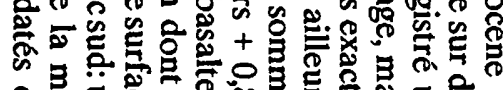

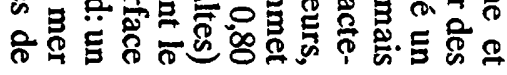

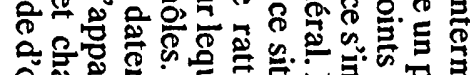

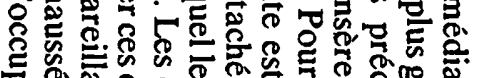
政学

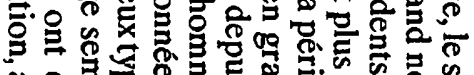

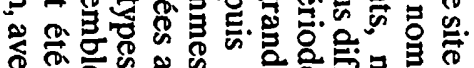
年

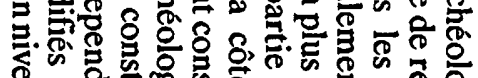

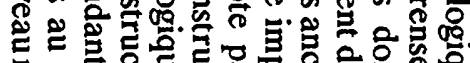

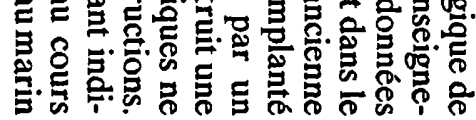

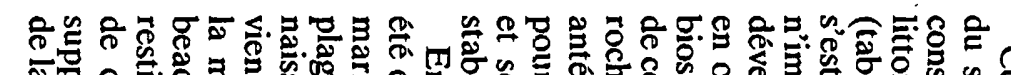

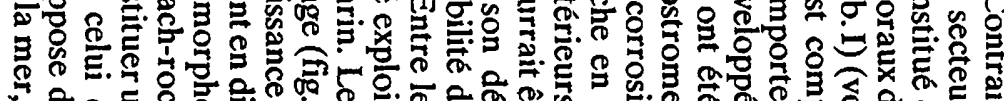

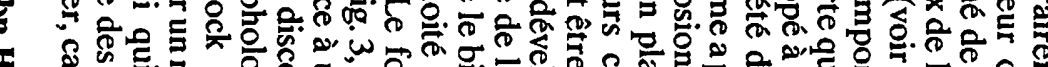

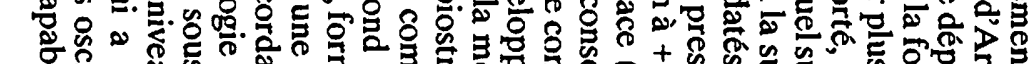

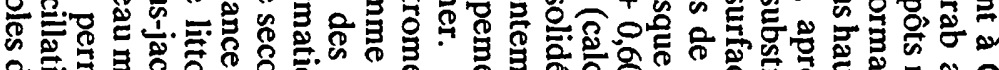

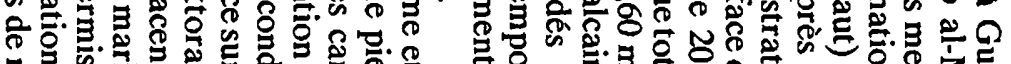

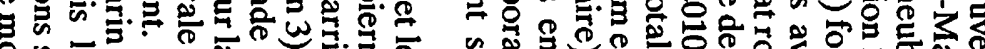
\%

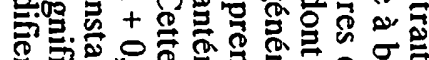

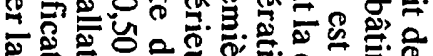

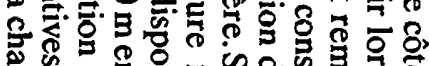

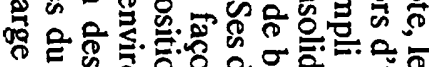

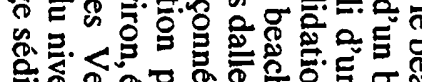

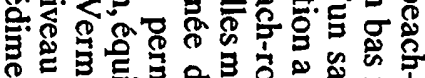

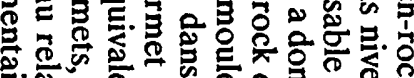
常.

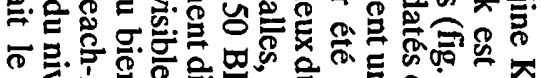

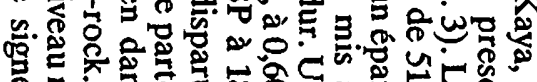

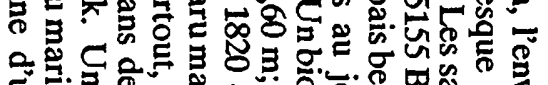

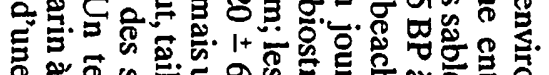
+ +

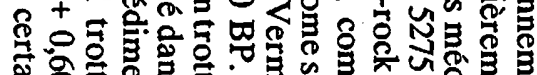

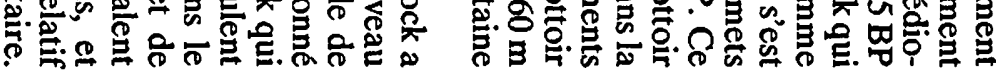

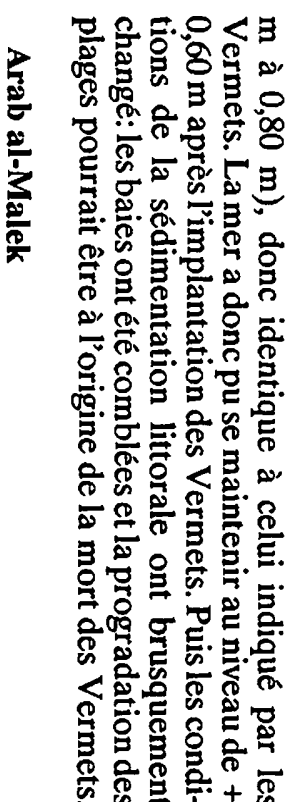

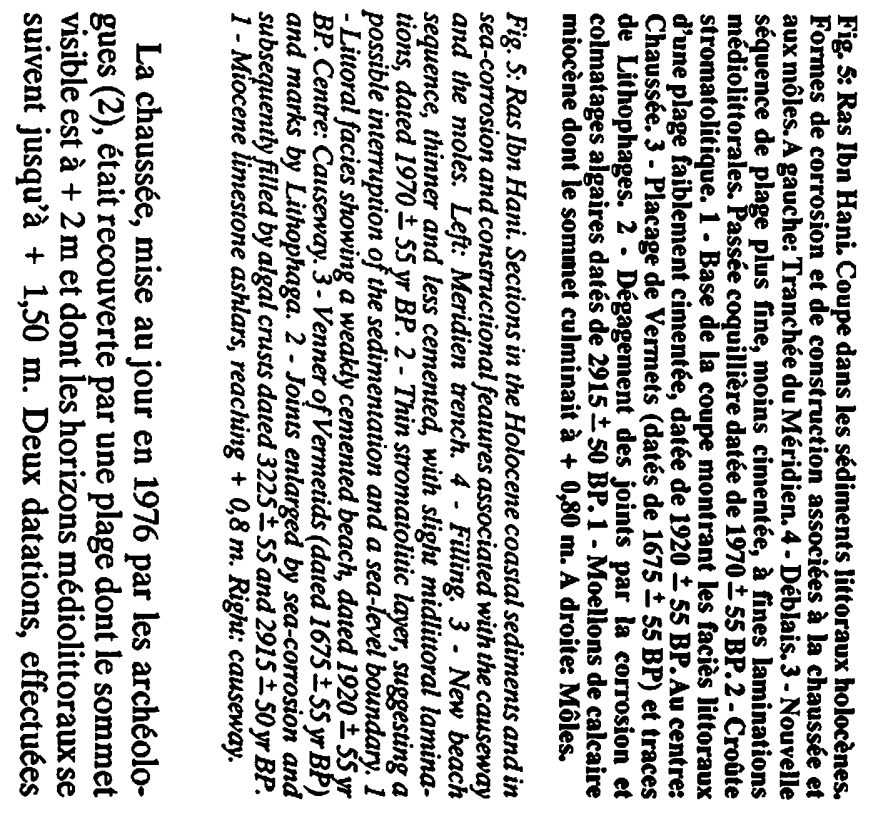

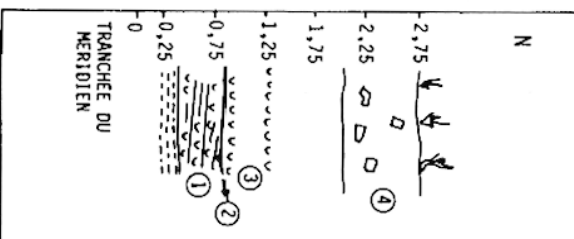

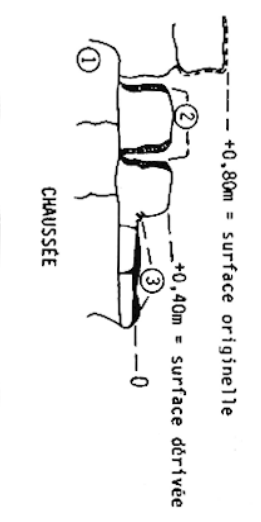

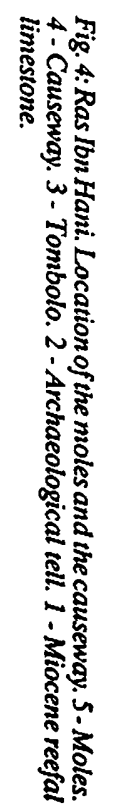

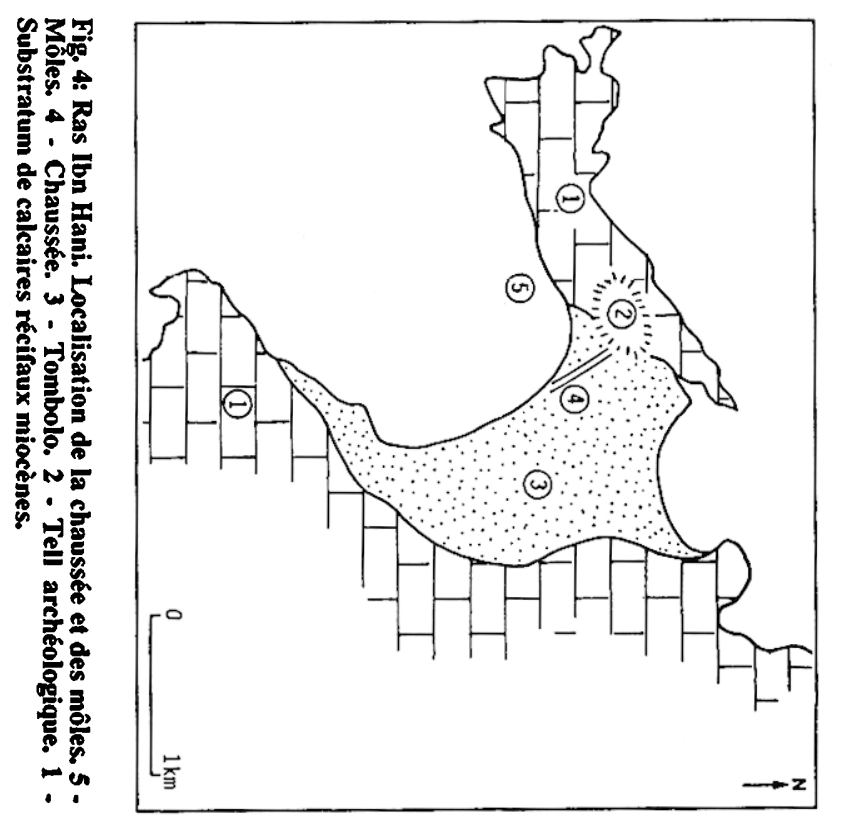


dans ces faciès aisément reconnaissables, à $+0,90 \mathrm{~m}$ et à $+0,45 \mathrm{~m}$, ont donné respectivement des âges de 1970 $\pm 55 \mathrm{BP}$ et de $1920 \pm 55 \mathrm{BP}$.

\section{DISCUSSION}

\section{L'ENCHAINEMENT GENERAL DES FAITS}

Les datations $14 \mathrm{C}$ (tab. I) déterminent un intervalle $5960 / 5155$ pour le niveau-repère de $+1,20 \mathrm{~m}$ et un intervalle $2010 / 1820$ pour le niveau-repère de $+0,60 \mathrm{~m}$.

La position altitudinale et le bon état de conservation des biostromes à Vermets pourraient être le résultat de deux pulsations tectoniques positives d'une ampleur de $0,60 \mathrm{~m}$ chacune à $6000 \mathrm{BP}$ et à $2000 \mathrm{BP}$.

On doit toutefois se poser la question suivante: les séquences sédimentaires observées dans chaque site sont-elles le résultat de variations réelles du niveau de la mer (quelle que soit l'origine de ce mouvement) ou sont-elles dues à de simples variations de la charge sédimentaire en transit?

\section{Le niveau de $+1,20 \mathrm{~m}$}

Les âges en années réelles des événements repérés à Guverdjine Kaya et à Arab al-Malek sont très proches les uns des autres et, s'ils ne se superposent pas, leur contiguiité est telle qu'ils ne sont pas séparables de manière irréfutable. L'interprétation est, de plus, rendue difficile par le fait que les supports des datations sont de nature et de signification éco-environnementale différentes.

L'épisode le plus ancien (4680-4360 av. J.-C.) correspond a priori à la formation deltaïque (fig. 3, [1]) d'Arab al-Malek. Celle-ci s'est mise en place lors du niveau marin de $+1,20 \mathrm{~m}$ et en fonction d'un trait de côte plus interne que l'actuel. Les datations sur les coquilles d'une formation sédimentaire de plage ont tendance à vieillir celle-ci (Flessa et al., 1993), notamment parce que l'âge calculé est celui de la mort des organismes. La mort des Vermets de Guverdjine Kaya pourrait être due à l'un des deux épisodes sédimentaires repérés à Arab alMalek, probablement au second (formation 2, datée sur coquilles de 3780 à 3500 av. J.-C).

Les blocs de Vermets contenus dans la formation supérieure [2] d'Arab al-Malek (5275 $\pm 65 \mathrm{BP}$, soit 39403630 av. J.-C.) traduisent une situation antérieure au dépôt 2 mais postérieure au dépôt 1 . Le littoral était alors constitué d'un substrat dur (beach-rock ou substratum) sur lequel les Vermets pouvaient se développer. Les Vermets d'Arab al-Malek ne seraient donc pas contemporains de ceux de Guverdjine Kaya (4330-3950 av. J.-C.).

Dans l'état actuel de nos connaissances, il n'y a pas lieu de relier ces multiples témoins de variations de la ligne de rivage à des niveaux marins différents. Une mer à la cote moyenne de $+1,20 \mathrm{~m}$ suffit à expliquer les événements. La côte syrienne connaissait alors une dynamique changeante: des alternances locales de progradation et de démaigrissement des plages (sous l'action d'apports fluviatiles très changeants qu'il reste à expliquer) fournissaient aux Vermets le substrat nécessaire à leur installation.

\section{Du niveau de $+1,20 \mathrm{~m}$ à celui de $+0,60 \mathrm{~m}$}

A Guverdjine Kaya, des lambeaux de sédiments marins coquilliers, restés accrochés à la falaise bien au-dessus (jusqu'à $+5 \mathrm{~m}$ ) du niveau à Vermets, ont été datés de $3590 \pm 70$ BP $(1820-1440$ av. J.-C. $)$ à $2945 \pm 55$ BP (970760 av. J.-C.). Déplacés par la dérive littorale, les fortes houles et les vents d'ouest, les sables et les coquilles ont pu être accumulés très haut le long de la paroi, que le niveau de la mer ait été à $+1,20 \mathrm{~m}$ ou à $+0,60 \mathrm{~m}$.

Le niveau relatif de la mer a probablement changé alors que ces dépôts étaient encore en place. En effet, à Ras Ibn Hani, un placage de Vermets prélevé à la racine des môles et deux fragments de sédiment induré dans les joints de la chaussée ont été datés respectivement de $2590 \pm 45$ BP (520-320 av. J.-C.), $3225 \pm 55$ BP (13701010 av. J.-C.) et $2915 \pm 50$ BP (910-750 av. J.-C.) (tab. I). Les Vermets fournissent ainsi à la fois un âge et une cote qui peuvent servir de repère: vers 500 av. J.-C., la mer se tenait à $+0,50 \pm 0,2 \mathrm{~m}$ au-dessus du niveau actuel.

La mer a pu passer à ce niveau brutalement (mouvement tectonique) ou bien s'abaisser progressivement (mouvement eustatique), abandonner des dépôts qui ont recouvert tout le littoral et remonter ensuite au niveau de $+0,60 \mathrm{~m}$. La mer a alors érodé les sédiments qu'elle avait précédemment déposés et atteint à nouveau le substratum calcaire et, comme à Guverdjine Kaya, le plan des falaises.

\section{Le niveau de $+0,60 \mathrm{~m}$}

Si ce niveau est atteint depuis 520 av. J.-C. (en fait entre 520 et 320 av. J.-C.), il n'est bien matérialisé, par un nuage de datations, qu'entre 150 et 650 ap. J.-C. Les datations et les altitudes sont cohérentes, même avec des supports différents (Vermets ou coquilles de Lamellibranches) et des types de dépôt variés (biostromes ou sédiments de plage). La mer aurait pu rester à ce niveau durant sept siècles au moins.

Mais, comme pour le niveau de $+1,20 \mathrm{~m}$, des datations s'écartent du schéma général: nous avons découvert à Ras al-Bassit des Vermets ayant un âge beaucoup plus récent (entre $1335 \mathrm{BP}$ et $1400 \mathrm{BP}$, soit entre 730 et 1100 ap. J.-C.) mais situés à la cote-repère de $+0,60 \mathrm{~m}$ et à Ras Ibn Hani des Vermets d'un âge très proche $(1675 \pm 55 \mathrm{BP}$, soit 550 à 770 ap. J.-C.) mais situés à un cote beaucoup plus basse (le niveau actuel).

\section{Du niveau de $+0,60 \mathrm{~m}$ à l'actuel}

La plage ayant recouvert la chaussée et les môles de Ras Ibn Hani a été mise en place vers 250-550 ap. J.-C. Les Vermets à la cote $+0,60 \mathrm{~m}$ ont été enfouis sous des sédiments coquilliers et sont morts. A Arab al-Malek, les Vermets ont également été fossilisés sous une plage (formation 3). Les deux épisodes ultimes de Ras Ibn Hani et de Arab al-Malek ont probablement la même signification: ils ont été mis en place entre 250 et 550 ap. J.-C. A Guverdjine Kaya, le beach-rock a fossilisé non seulement les Vermets inférieurs mais également le biostrome supérieur (mort depuis longtemps) et les formes de corrosion (cavités, encoche et trottoir).

Une nouvelle tendance au colmatage a donc gagné l'ensemble du littoral syrien. Mais ni la durée ni la cause ne sont connues: mouvement tectonique brutal ou apports

(2) Les fouilles archéologiques du site de Ras Ibn Hani sont menées dans le cadre d'une collaboration entre le Ministère Français des Affaires Ėtrangères et la Direction Générale des Antiquités et Musées de Syrie. Elles sont dirigées conjointement par H. Bounni et J. et E. Lagarce, qui ont bien voulu nous héberger dans leur maison de fouilles et que nous tenons à remercier ici. 
fluviatiles massifs.

Les Vermets plaqués sur le flanc sud de la chaussée de $R$ as Ibn Hani à une cote proche de celle du niveau marin actuel ont un âge de $1675 \pm 55 \mathrm{BP}$, soit de 550 à 770 ap. J.-C. On pourrait donc considérer que la mer a atteint son niveau actuel dès le VIème siècle ap. J.-C.

Sur le flanc nord du cap de Ras al-Bassit, des Vermets montrent qu'en fait le niveau de la mer s'est élevé ensuite pour atteindre à nouveau $+0,60 \mathrm{~m}$ vers le Xème siècle. Toutefois, cet épisode n'est pas attesté par la présence de sédiments. Parmi les nombreuses datations de ce niveau, non seulement en Syrie mais également en Turquie et au Liban, aucune autre datation n'est postérieure au VIème siècle. Ce deuxième épisode à $+0,60$ $\mathbf{m}$ pourrait donc avoir été très bref ou très limité géographiquement. Par ailleurs, aucun élément ne permet de savoir - du moins à Ras al-Bassit - depuis combien de temps exactement la mer est revenue à son niveau actuel.

\section{LA COMPARAISON AVEC DES LITTORAUX VOISINS}

\section{La Turquie}

Au débouché de l'Oronte (Nahr al-Assi), deux niveaux de Vermets ont été reconnus (Pirazzoli et al., 1991). Le niveau supérieur, situé à $+2 \mathrm{~m}$ au sud du delta, mais incliné entre $+2,5 \mathrm{~m}$ et $+3 \mathrm{~m}$ plus au nord, a été daté entre environ 5570 et 3000 BP (3). Le niveau inférieur, situé entre $+0,7 \mathrm{~m}$ et $+0,8 \mathrm{~m}$, a été daté entre 3300 et 1750 BP. Ici, les changements de niveau de la mer se seraient produits brusquement, à deux reprises, vers $3000 \mathrm{BP}$ et au VIème siècle de notre ère. Cette partie du littoral turc ne semble pas avoir connu une sédimentation comparable à celle de la Syrie.

\section{Le Liban}

Au sud, le littoral libanais montre un certain nombre de témoins de variations récentes de la ligne de rivage: des trottoirs de corrosion et des encoches étagés, des biostromes à Vermets plus ou moins épais ancrés sur la roche calcaire elle-même mais aussi sur des conglomérats holocènes, notamment dans l'archipel de Tripoli (Sanlaville, 1977). Le Zennadien est un épisode de haut niveau marqué par des Vermets à $+2,20 \mathrm{~m}$ datés de $3490 \pm 80 \mathrm{BP}$ (soit de -2035 à - $1632 \mathrm{BC}$ ). Le Tabarjien est un épisode moins élevé (vers $+0,80 \mathrm{~m}$ ) daté par des Vermets de 2035 BP à 1880 BP.

En Turquie, au débouché de l'Oronte, comme au Liban, les événements paraissent beaucoup plus nets qu'en Syrie, puisque seuls les deux rivages émergés sont bien marqués, par des Vermets mais également par des trottoirs de corrosion et des encoches. Le passage d'un niveau à l'autre s'est fait rapidement, comme l'atteste l'absence de placages de Vermets dans la tranche intermédiaire, et il est d'origine tectonique. Cependant, l'ampleur des pulsations recensées au Liban n'est pas comparable à ce qui a été observé sur le littoral syrien.

\section{CONCLUSION}

La Syrie présente un enchaînement d'événements littoraux dans lequel sont étroitement associés des pro- cessus de corrosion marine (trottoirs, encoches), de sédimentation (plages, dépôts deltaïques), de diagenèse carbonatée précoce (beach-rock) et de construction organogène (biostromes à Vermets). Chaque processus a son rythme particulier de mise en place, plus ou moins long, et il est porteur d'enseignements qui lui sont propres, notamment dans la définition altitudinale du niveau marin dont il est la manifestation.

Si les témoins des phases de sédimentation intense sont mal conservés dans les secteurs rocheux, parce qu'il s'agit de falaises dont le façonnement a été polygénique et polychronique, ils envahissent au contraire les secteurs bas qu'ils remblaient d'épais dépôts fluviatiles et marins. Au pied des falaises, la lecture des événements s'effectue verticalement. Le long des plaines, c'est au contraire une succession horizontale de faits qu'il faut saisir, les témoins des épisodes les plus externes ayant disparu sous l'action du recul de la ligne de rivage.

En Syrie, les deux niveaux marins s'individualisent nettement $\mathbf{a}+1,20 \mathrm{~m}$ et $+0,60 \mathrm{~m}$ et un relais constant s'établit dans le temps et dans l'espace, le long de la côte, entre processus d'érosion et processus de construction (fig. 6). Entre 6000 BP et aujourd'hui, le littoral syrien a connu plusieurs phases de progradation et plusieurs phases d'érosion qu'il faut essayer d'expliquer.

La variation générale de la ligne de rivage dans les six mille dernières années est probablement tectonique et on pourrait imaginer que le relèvement du continent $a$ pu entraîner, dans le volume montagneux tout proche, une légère reprise du creusement des cours d'eau et une déstabilisation des nombreuses terrasses pléistocènes qui expliqueraient les différents épisodes sédimentaires. Mais il est moins sûr que les mouvements aient été brusques (instantanés), à l'exception du dernier sursaut $\grave{a}+0,60 \mathrm{~m}$ (Ras al-Bassit). Quand on prend en compte tous les indices de datation et de niveaux possibles, les cimentations inter-joints de la chaussée et des môles (tab. I) par exemple, tout se passe comme si le littoral n'avait enregistré qu'un lent mouvement de surrection.

En tout cas, les épisodes de colmatage gomment un certain nombre de phénomènes. Les modifications du tracé littoral en substrat meuble sont assez lents, car il faut un certain temps aux sédiments pour se mettre en place puis être enlevés, en un endroit donné, et la dynamique littorale participe d'un effet d'onde sudnord qui augmente les temps de réponse. Il est actuellement difficile de dire quand et en combien de temps le niveau marin relatif est passé de $+1,20 \mathrm{~m}$ à $+0,60 \mathrm{~m}$ le long du littoral syrien: tous les secteurs ne sont pas en phase les uns avec les autres.

La période étudiée correspond à l'augmentation de l'emprise humaine, avec ses côtés néfastes (déforestation intense) et ses côtés bénéfiques (construction de terrasses de culture), se relayant dans le temps et dans l'espace selon les modalités de l'occupation. La cause des différentes phases d'alluvionnement reconnues sur le littoral syrien est probablement, pour une part notable, d'origine humaine.

Une origine climatique n'est cependant pas à écarter. Des éléments disparates font penser qu'une période plus humide a existé vers 4000-3500 BP dans les Cyclades mais aussi au Moyen-Orient. Les influences méditerranéennes humides de secteur ouest ont ainsi pu jouer un rôle non négligeable sur la façade occidentale de la montagne syrienne.

Enfin, le télescopage des événements rend difficile 
l'individualisation de chacun d'entre eux pendant une période aussi courte et dans une région où le niveau marin était proche de l'actuel.

\section{BIBLIOGRAPHIE}

DALONGEVILLE, $R, 1983$ - Variations de la ligne de rivage en Méditerranéé orientale au cours de l'Holocène: témoins morphologiques et archéologiques. Actes du colloque Les Cyclades antiques, 1982, Dijon, C.N.R.S., Lyon, 89-98.

DALONGEVILLE, R. et SANLAVILLE, $P_{,}, 1977$ - Témoins de lignes de rivage holocènes en Turquie méridionale. Bul. A.F.E.Q., 4, 53, 79-81.

DALONGEVILLE, R. et SANLAVILLE, P., 1979 - Les rivages holocènes de Turquie méridionale. Bul. Lab. Rhod. Géomorph., 4-5, 515 .

DALONGEVILLE, R. et SANLAVILLE, $P_{\text {. }} 1980$ - Les changements de la ligne de rivage en Méditerranée orientale, à l'époque historique. Exemple de la côte levantine. Actes du colloque Salamine de Chypre, Histoire et Archéologie, 13-17 mars 1978, Lyon, 19-32.

DALONGEVILLE, R et SANLAVILLE, $P_{.}, 1984$ - Essai de synthèse sur le beach-rock. In: Le beach-rock, Actes du colloque de Lyon, nov. 1983, Dalongeville édit., T.M.O. 8, Maison de l'Orient, Lyon, 161-167.

FLESSA, K.W, CUTLER, A.H. et MELDAHL, K.H., 1993 (sous presse) - Time and taphonomy: quantitative estimates of time- averaging and stratigraphic disorder in a shallow marine habitat. Paleobiology, 19, 29 p.

KELLETAT, D. and KAYAN, I., 1983 - Alanya batisindaki kiyilarda ilk 14C tatihlendirmelerinin Gec Holosen tektonik hareketleri. Türk. Jeol. Kurumu Bül., C, 26, 83-87.

PIRAZZOLI, P.A., 1986 - The Early Byzantine tectonic paroxysm. $Z$. Geomorphol., suppl. 62, 31-49.

PIRAZZOLI, P.A, LABOREL, J, SALIEGE, O, EROL,O, KAYAN, I. et PERSON, A, 1991 - Holocene raised shorelines on the Hatay coasts (Turkey): palaecological and tectonic implications. Mar. Geol., 96, 295-311.

SANLAVILLE, P, 1976 -Sur l'existence en Syrie de lignes de rivage holocènes supérieures à l'actuelle. Bul. Lab. Rhod. Géomorphol.,1, Bron, 45-52.

SANLAVILLE, P., 1977 - Etude géomorphologique de la région littorale du Liban, Thèse doctorat d'Etat, Brest, Publ. Univ. Libanaise, Beyrouth, 2 vol., 859 p.

SANLAVILLE, $P_{y}, 1978$ - Note sur la géomorphologie de la presqu'île d'Ibn Hani (Syrie). Rapport préliminaire sur la deuxième campagne de fouilles (1976) à Ibn Hani (Syrie). Syria, LV, 3-4, Paul Geuthner, Paris, 303-305.

STIROS, S.C., ARNOLD, M., PIRAZZOLI, PA, LABOREL, J., LABOREL, F. et PAPAGEORGIOU, S., 1991 - Historical coseismic uplift in Euboea Island (Greece). Earth and Planetary Sc. Lett., 108, 109-117.

STUIVER, M., PEARSON, G.W. et BRAZIUNAS, T., 1986 - Radiocarbon age calibration of marine samples back to 9000 cal Yr BP. Radiocarbon, 28, 2B, 980-1021. 